\title{
DEFINING GEOSPATIAL DATA FUSION METHODS BASED ON TOPOLOGICAL RELATIONSHIPS
}

\author{
D. S. Ahn, J. H. Park, J. Y. Lee**
}

Dept. of Geoinformatics, University of Seoul, Dongdaemun-gu, Seoul, 163 Seoulsiripdearo - \{ads903, junho7507, jlee @uos.ac.kr $\}$

KEY WORDS: Geospatial Data Fusion, Topological Data Model, 3D GIS, Anchor Node, IndoorGML

\begin{abstract}
:
Currently, geospatial datasets are produced in various models and formats in accordance with the spatial scale of the real world such as ground/ surface/underground or indoor/outdoor. The location-based services application also uses the optimal data model and format for each purpose. Therefore, there are various geospatial dataset for representing features of the same space. Various geospatial data on same object cause problems with the financial problems and the suitability of the data. In the paper, we reviewed how to integrate existing geospatial data to utilize geospatial data constructed in different models and formats. There are four main ways to fuse existing geospatial information. The existing geospatial data fusion methods consist of a method through geometry data conversion, a method through the aspect of visualization, a method based on attribute data, and a method using topological relationships. Based on this review, we defined a geospatial data fusion method on topological relationships, which is a method considering topological relationship between geospatial objects. In this method, the topological relationship of objects uses the basic concept of IndoorGML.
\end{abstract}

\section{INTRODUCTION}

The real world consists of a combination of geographic objects, which is an object connected to on space. However geospatial dataset is constructed and used individually according to the space scale of the real world such as ground / surface / underground or indoor / outdoor according to the purpose of geospatial dataset used for location-based services, as seen in Figure 1.

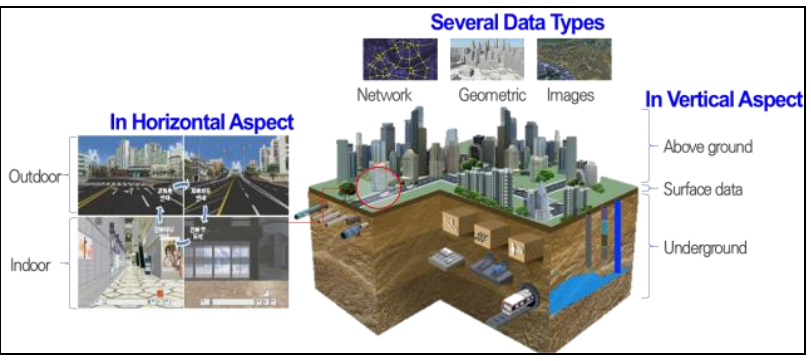

Figure 1. Various geospatial datasets according to representing the real world

In addition, geospatial data has problems that occurs in various data formats, the connectivity of two-dimensional and threedimensional data, and consistency representation in geometric and topological data. In order to provide seamless LBSs between indoor and outdoor spaces, one of the most important requirements is to connect indoor to outdoor spaces (Lee et al. 2014). In general, while geographical features in outdoor space have been represented as two-dimensional objects, spatial entities in micro built-environments such as buildings have been represented as 3D objects in BIM or CityGML datasets. These two-dimensional and three-dimensional geospatial data use various data models and formats depending on the generation method. According to the purpose of the LBSs, as the indoor geospatial data and the outdoor geospatial data are constructed, managed, and utilized, problems arises in the operation of the geospatial system, compatibility of the geospatial data and connectivity.

In order to solve these problems, researches have been conducted to fuse geospatial data generated by various construction methods and data models (M. Gunduz et al. 2016). As the developed methods to improve the interoperability and the compatibility of data by combining the spatial data and the data of different formats, there are four approaches including a data fusion method using geometric data conversion, a method through the aspect of visualization, a data fusion method based on attribute data, and recently proposed geospatial data fusion methods based on topological relations of spatial objects. In the following section, we describe the existing spatial data fusion methods. And we present the proposed geospatial data fusion method using topological relations between geospatial object based on IndoorGML.

\section{DEFINING GEOSPATIAL DATA FUSION METHODS BASED ON TOPOLOGICAL RELATIONSHIPS}

Conventional geospatial data has limitations such as various formats and lack of connectivity between object. To solve the limitations, geospatial data fusion method has been studied. The existing geospatial data fusion research was conducted in three ways.

The first geospatial data fusion method is data fusion method through geometric data transformation. Data fusion method through geometric data transformation is actively studied for exchanging BIM data and CityGML dataset (Tang et al. 2015). As shown in Figure 2, CityGML data and BIM data is international standard data model to manage and utilize indoor and outdoor geospatial data.

\footnotetext{
* Corresponding author
} 


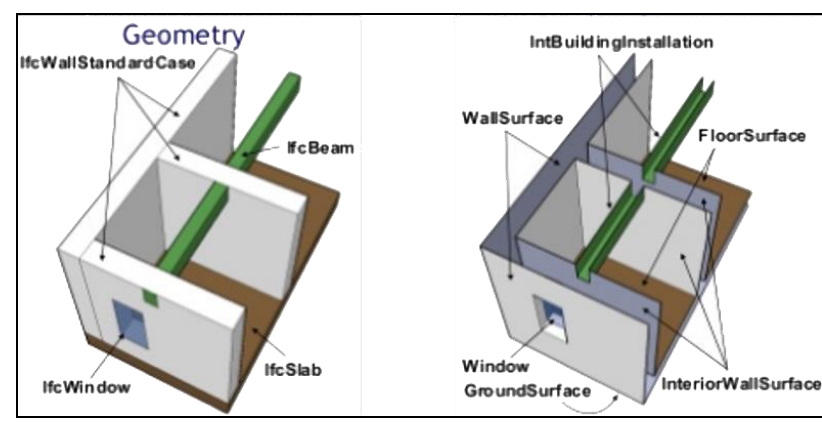

Figure 2. BIM data and CityGML data (Thomas H Kolbe, 2010)

Research for the conversion of information between two standards is continuing to manage and utilize indoor geospatial data. This method can visually represent different geospatial dataset in one application system. However, it is necessary to analyse the object of geospatial data and correspond to the target data. There are also geospatial dataset that can't be converted, such as image data.

Secondly, there is a data fusion method through the aspect of visualization. The model representing the space visualizes other data in one application system. For example, V-world that is represent as an open space information system in Korea, digital elevation model and orthographic image to visually link ground geospatial and indoor geospatial data.

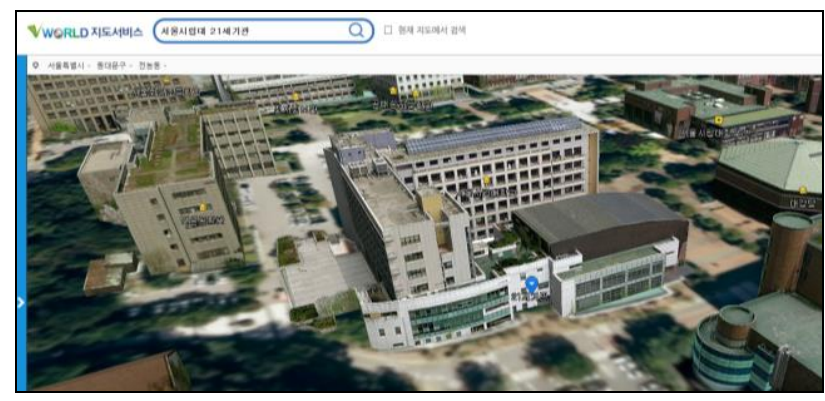

Figure 3. Example of the aspect of Visualization(V-world, 2017)

This method is very useful in applications because it is visually linked. However, the visualization side method requires precise coordinate information of the geometric objects. Geometry objects should also be represented in the same coordinate system. If these conditions are not met, the topological relationship of the objects is unknown.

The third approach is a data fusion method based on attribute data. This method associates the attribute information of the object with another dataset using a primary key. Each object must have the same coordinate system and attribute values of the same attribute field. Objects must also be spatially close together ( $\mathrm{Yu}$ et al. 2014). The method based on attribute data can fuse various information through connection of each attribute data. On the other hand, if objects do not have the same coordinate system and are not close to each other in space, they can't be fused.

Finally, there is a data fusion method based on topological relationships among spatial entities. This method is a method of merging data by connecting topological relationship data models of different data sets. The Topological Relation Method (TRM) has been proposed using topological relationships between spatial objects to directly use various geometric data generated by different data models for indoor spatial location services (Lee et al. 2014). As shown Figure 4, data fusion between IFC and CityGML, which are different data formats, was performed by using topological representations using a Node-Link graph (Ihab et al. 2011).

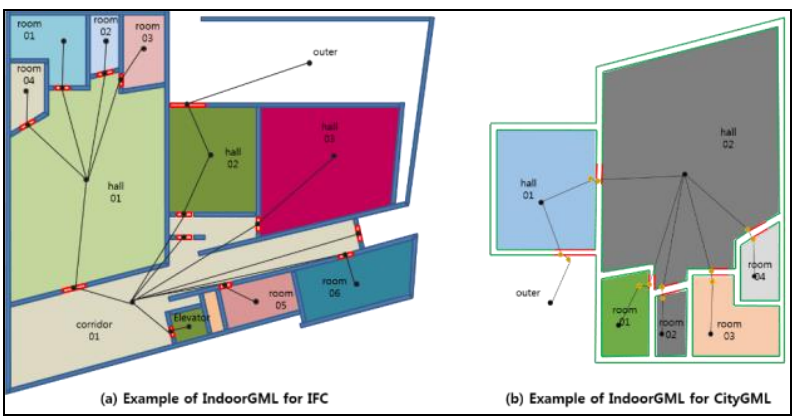

Figure 4. Node-Link graph in IFC and CityGML

The same type of networked-based topological representations utilizing node-link graphs are generated from the two geometry models by defining the topology data generation procedure and necessary attribute information for each geometry model. As shown Figure 5, IndoorGML proposed an additional element for connecting indoor and outdoor spaces, which is presented as an anchor node concept. In all indoor spaces, there is an entrance for outdoor use, which is used as anchor node to connect indoor and outdoor (OGC, IndoorGML v1.0, 2014).

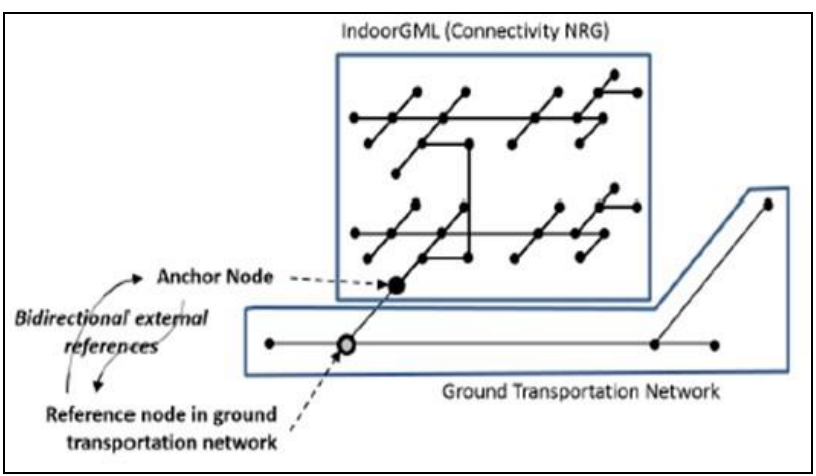

Figure 5. Anchor node connecting indoor and outdoor space networks (OGC, 2014)

In this way, the developed methods for fusing geospatial data are composite methods through geometric data transformation, methods based on attribute data, method based on topological relationships among spatial entities. However, the data fusion methods with geometric transformation requires the relationship of the object data and target data. The method based on attribute data has limitation that can't represent a topological relationship between objects without accurate geometric information. The data fusion method based on topological relationship models for connecting indoor and outdoor using AnchorNode proposed by IndoorGML was presented in the conceptual level, whose model has limitations in implementing a practical integrated system to provide seamless LBS services in real world.

\section{CONCLUSION}

The geospatial dataset is constructed in the same way as the real world such as ground / surface / underground or indoor / 
outdoor. These geospatial dataset are composed of various models and formats according to the space scale and the purpose of geospatial data used for location-based services. To solve this problem, we reviewed the method of merging existing geospatial data.

The existing geospatial data fusion methods consist of a method through geometric data conversion, a method through the aspect of visualization, a method based on attribute data, and a method using topological relationships. The method through geometric data conversion can represent different geospatial information dataset in one application system. However, the conversion data needs to be associated with the format of the target data and conversion such as image data is sometimes impossible. The method though aspect of visualization is useful in application. However, this method needs precise coordinate information of geometric objects to know the topological relationships of objects. The method based on attribute data can fuse various information through connection using the primary key, but it can not represent relation between objects without accurate geometric information. The method based on topological relationship among spatial entities can fuse geospatial data without conversion of original data. However, the topological relationship between indoor and outdoor remains at a conceptual level.

\section{ACKNOWLEDGEMENTS}

This research was supported by a grant (17NSIP-B135746-01) from National Spatial Information Research Program (NSIP) funded by Ministry of Land, Infrastructure and Transport of Korean government.

\section{REFERENCES}

H. M. Yu, J. Y. Lee, 2014. Developing a Method for Attribute Data Integration of Spatial Data, dongdaemun-gu, Seoul, Master's thesis, University of Seoul, Korea, pp.6-12.

Ihab. H., Manfred. E., Zlatanova. S et al., Initial Investigations for Modelling Interior Utilities Within 3D Geo Context: Transforming IFC-Interior Utility to CityGML/UtilityNetworkADE, In: Advances in $3 D$ GeoInformation Sciences, pp. 95-113.

J. Y. Lee., H. Y. Kang., and Y. J. Kim., 2014. Developing Data Fusion Method for Indoor Space Modelling based on IndoorGML Core Module. In:Journal of Korea Spatial Information Society, Vol.XX/W2, pp. 31-44,

J. Y. Lee, A Three-Dimensional Navigable Data Model to Support Emergency Response in Microspatial BuiltEnvironments, In: Annals of the Association of American Geographers, Vol XC-VII/W3, pp. 512-529.

K. J. Li., J. Y. Lee., 2013. Basic Concepts of Indoor Spatial Information Candidate Standard IndoorGML and its Applications , In: Journal of Korea Spatial Information Society Vol XXI/W3, pp.1-10.

M. Gunduz., U. Isikdag., M. Basaraner, 2016, A REVIEW OF RECENT RESEARCH IN INDOOR MODELLING \& MAPPING. In: International Archives of the Photogrammetry,
Remote Sensing and Spatial Information Sciences (ISPRS), Vol XLI-B4, pp. 289-294.

Tang S. J., Zhu, Q., Wang, W. et al., Automatic Topology Derivation From Ifc Building Model for in-Door Intelligent Navigation, In: The International Archives of Photogrammetry, Remote Sensing and Spatial Information Sciences, Vol XL/W4, pp. 7-11.

Revised August 2018 